\title{
Butterfly-shaped pigment dystrophy
}

INSERM

\section{Source}

INSERM. (1999). Orphanet: an online rare disease and orphan drug data base. Butterflyshaped pigment dystrophy. ORPHA:99001

Butterfly-shaped pigment dystrophy is a patterned dystrophy of the retinal pigment epithelium (see this term) characterized by abnormal accumulation of lipofuscin in a butterfly-shaped distribution at the retinal pigment epithelium level. Patients manifest with a slowly progressive loss of vision that often only becomes apparent in old age. 